\title{
La biblioteca integrada en la enseñanza universitaria online: situación en España
}

\section{Integrating libraries into online university learning: the current situation in Spain}

\author{
Natalia Arroyo-Vázquez; José-Antonio Gómez-Hernández
}

Cómo citar este artículo:

Arroyo-Vázquez, Natalia; Gómez-Hernández, José-Antonio (2020). “La biblioteca integrada en la enseñanza universitaria online: situación en España”. Profesional de la información, v. 29, n. 4, e290404.

https://doi.org/10.3145/epi.2020.jul.04

Artículo recibido el 04-06-2020

Aceptación definitiva: $14-06-2020$

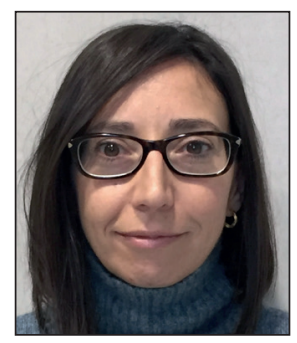

Natalia Arroyo-Vázquez https://orcid.org/0000-0002-4692-3420

Universidad de Navarra Servicio de Bibliotecas. Biblioteca Central Campus Universitario. 31009 Pamplona, España natalia.arroyo@gmail.com

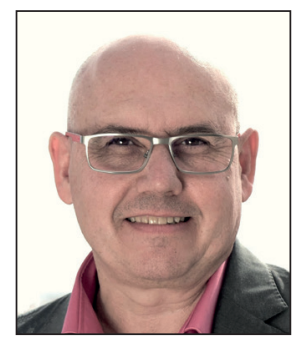
José-Antonio Gómez-Hernández https://orcid.org/0000-0003-4532-1142

Universidad de Murcia Facultad de Comunicación y Documentación Campus de Espinardo, Edificio 3 30100 Murcia, España jgomez@um.es

\section{Resumen}

Las bibliotecas universitarias españolas se han visto obligadas a adaptar sus servicios al entorno virtual tras el cierre de las aulas en marzo de 2020 con motivo del estado de alarma decretado a causa de la Covid-19. Ante la perspectiva de un nuevo curso con un mayor componente digital en la docencia, estos servicios necesitarán fortalecerse. En este artículo se analiza qué acciones están desarrollando las bibliotecas universitarias para reforzar el apoyo a la enseñanza online y su integración en el campus virtual, así como las posibles mejoras y limitaciones que encuentran. Para ello se ha consultado a una selección de profesionales de bibliotecas universitarias y se ha revisado además la bibliografía en busca de experiencias en otros países que puedan servir como referente. Tanto la bibliografía como las personas consultadas coinciden en dos aspectos: la necesidad de colaboración con otros departamentos de la universidad y el amplio margen de mejora posible en la integración de la biblioteca en la enseñanza online, que en la actualidad es escasa.

\section{Palabras clave}

Bibliotecas universitarias; Apoyo a la docencia; Enseñanza online; Bibliotecarios integrados; Campus virtual; Plataforma de enseñanza virtual; Covid-19; Bibliotecas; Colecciones digitales; Servicios digitales; España.

\begin{abstract}
Spanish university libraries have had to adapt their services to the virtual environment after the classroom-based teaching lockdown in March 2020 due to Covid-19. Given the possibility of a greater virtual dimension of learning and teaching during the next academic year, these library services should be improved. This paper analyzes how university libraries are strengthening their support for online learning and are progressively being integrated into the virtual campus, as well as the actions that are necessary to enhance these services and the main difficulties perceived by their librarians. For this purpose, a sample of professionals from university libraries was interviewed, and the research literature on this subject was also reviewed to identify the main international experiences that can be considered as benchmarks for improving virtual library services. Both the research literature and the librarians interviewed agree on two aspects: the need to increase collaboration with other university departments and the wide scope for improvement in the integration of the library into online learning, which is currently limited.
\end{abstract}

Agradecimiento

Agradecemos a las bibliotecarias y bibliotecarios de las universidades que respondieron a nuestra entrevista sus valiosas consideraciones. 


\section{Keywords}

University libraries; Online learning; Embedded librarians; Virtual campus; Learning management system; Covid-19; Libraries; Digital collections; Digital services; Spain.

\section{Obligada adaptación de las bibliotecas a la enseñanza online}

Durante la etapa de confinamiento por la Covid-19 las universidades han tenido que continuar el curso académico de forma no presencial ante el cierre de las aulas en el mes de marzo de 2020. Como consecuencia, el profesorado ha debido acomodar -en algunos casos improvisar- de un día para otro su metodología de enseñanza y llevarla al campus virtual. Todo apunta a que esta situación no será transitoria, sino permanente, con clases de aforo reducido y facilitando el seguimiento a quienes no puedan asistir al aula. En opinión de Dans (2020),

"en el futuro, toda actividad educativa tendrá lugar no en modo presencial u online, sino en modo líquido, capaz de trasladarse de uno a otro soporte de manera inmediata".

Pero esto no resta importancia a la presencialidad de la enseñanza, que resulta insustituible.

A su vez, las bibliotecas universitarias han cerrado sus puertas, con gran pesar de muchos usuarios. Pero han seguido trabajando con el personal en sus hogares. De acuerdo con los datos recopilados por Rebiun (2020a), en un primer momento se han mantenido los servicios no presenciales y la atención al usuario por teléfono, correo electrónico y mensajería. Los períodos de préstamo se han ampliado automáticamente y se han incrementado los esfuerzos en la difusión de las colecciones electrónicas.

Ante una situación imprevista y que en principio se percibía como provisional, las bibliotecas se han ido adaptando poco a poco a la nueva coyuntura y han buscado formas de reanudar la misma actividad en el entorno virtual. Pero, ¿en qué medida están las bibliotecas preparadas para dar soporte a la enseñanza online a medio y largo plazo? ¿De qué forma se podría mejorar este apoyo? ¿Qué estrategias se podrían mantener a largo plazo para afianzar los servicios online?

Para responder a estas cuestiones se ha revisado la bibliografía sobre el papel de las bibliotecas en la enseñanza online, con el fin de conocer las experiencias que se han compartido a nivel internacional, y se ha entrevistado a una muestra diversa de profesionales de las bibliotecas universitarias españolas con el objetivo de conocer la situación en la que se encuentran sus bibliotecas y las acciones que están desarrollando como apoyo a la docencia online y, especialmente, en qué medida están integradas las bibliotecas en el campus virtual de la universidad.

\section{Integración de la biblioteca universitaria en la enseñanza online: estado de la cuestión}

Si el campus virtual es el espacio que visitan los estudiantes de las titulaciones que imparten docencia online, la biblioteca corre el riesgo de pasar desapercibida si no logra integrarse en él. Para Kampa (2007), no basta con desarrollar la presencia virtual paralelamente, en espacios propios de la biblioteca: para que la biblioteca sea relevante para alumnos y profesores su presencia debe estar en el propio campus virtual.

Para ello es esencial lograr alianzas con los agentes o stakeholders involucrados en la enseñanza online, como son los profesores y el departamento encargado de la administración de la plataforma educativa de la universidad. Se trata de un aspecto en el que existe amplio consenso y que se repite en la bibliografía hasta convertirlo en obviedad. Pero también es uno de los puntos más difíciles de implementar en muchas universidades, pues lo cierto es que no es habitual tener en cuenta a la biblioteca en la docencia online, lo que hace necesario establecer contactos previos para conseguir su integración y evidenciar sus beneficios (Piña, 2007). Además, es esencial conseguir una alianza con el profesorado, pues se obtiene una mayor atención del alumnado cuando son los docentes quienes recomiendan los recursos de la biblioteca (Farkas, 2015).

La Association of College \& Research Libraries (ACRL, 2016) publicó los estándares para los servicios bibliotecarios para la enseñanza a distancia, en los que se planteaba la necesidad de garantizar los mismos derechos para los estudiantes de titulaciones a distancia que aquellos que tienen quienes acuden a clase presencialmente. También establecen la figura del bibliotecario de educación a distancia (distance learning librarian), especializado en los servicios bibliotecarios para esta modalidad de enseñanza, reconociendo así la necesidad de invertir recursos.

Respecto a la integración de la biblioteca en el campus virtual, Shank y Dewald (2003) identifican dos niveles: macro, que consiste en establecer acciones generales que se puedan incluir en todas las asignaturas, y micro, que supone un mayor nivel de personalización, teniendo en cuenta que los recursos de interés varían en función de las materias de estudio. Además, los autores de este artículo consideramos que la integración puede referirse a dos aspectos:

- Integración de los recursos, que consistiría en incrustar recursos de la biblioteca dentro del propio campus virtual o en incluir enlaces a ellos.

- Integración del personal, de manera que los bibliotecarios participen activamente o como apoyo en los procesos de aprendizaje.

La integración técnica de los recursos de la biblioteca comprende actuaciones de lo más variado, que pueden ir desde algo tan sencillo como incluir enlaces a la web y a los recursos de la biblioteca en el campus virtual hasta proporcionar al 
profesorado un conjunto de objetos de aprendizaje, basados en recursos de la biblioteca, que pueden utilizar a la hora de diseñar los materiales de la asignatura. En este sentido, los recursos de utilidad para las asignaturas son también variados: bases de datos especializadas y herramientas de búsqueda, bibliografía recomendada, guías temáticas o servicios de referencia, por citar algunos. La integración puede plantear barreras técnicas, que requieren la comunicación entre el software que se emplea en el campus virtual.

Por otra parte, la integración del personal bibliotecario en las asignaturas se puede concretar de múltiples formas: mediante asistencia a través de referencia virtual, participando en foros de discusión, facilitando ayuda al profesorado en lo relacionado con los recursos de la biblioteca e incluso participando directamente en la formación en alfabetización informacional o en la creación de recursos de aprendizaje. En este sentido se utilizan en español la expresión «bibliotecario integrado» y otras que parecen más forzadas, como «incrustado» o «embebido», para referirse a lo que en el ámbito anglosajón se conoce como embedded librarian. No se trata de una figura exclusiva en el apoyo a la formación online, sino que se emplea también en el apoyo a la investigación (Torres-Salinas, 2011) o en otras tareas, como la adquisición de la bibliografía recomendada y la formación en competencias transversales (Martínez-Trujillo, 2013).

Los mencionados estándares sobre los servicios bibliotecarios para la enseñanza a distancia de la ACRL (2016) definen la figura del embedded librarian como aquel que «participa activamente junto con el profesor de las asignaturas en la docencia y que se identifica y se hace accesible en el sistema de gestión de las asignaturas». Este término parte de la figura del bibliotecario enlace (liaison librarian), que nace en Estados Unidos en los años cuarenta como apoyo en el desarrollo de las colecciones y cuyas funciones se han ido ampliando con el tiempo, como la formación, elaboración de guías temáticas o la asistencia a las funciones de docencia e investigación (Henry, 2014).

EI NMC Horizon Report: 2014 library edition (Johnson et al., 2014), citado por Cervera-Farré et al. (2015), señala entre las funciones del subject liaison librarian la

"colaboración con los académicos en la elaboración de material didáctico para los cursos, formación en competencias informacionales a profesores y estudiantes, desarrollo de colecciones especializadas de apoyo a los planes docentes y asesoramiento de los docentes en los recursos de información de sus disciplinas o asignaturas".

Entre las buenas prácticas para estos bibliotecarios integrados se pueden identificar las siguientes (York; Vance, 2009; Hoffman; Ramin, 2010):

- Conocer el campus virtual.

- Establecer un enlace con los administradores del campus y con el profesorado.

- Involucrarse directamente en las asignaturas y participar de forma activa en ellas.

- Contar con la ayuda de otros bibliotecarios ante el riesgo de sobrecarga de tareas.

- Crear contenidos y recursos de formación en competencias informacionales.

- Establecer estrategias para seleccionar las asignaturas en las que se está presente y establecer un tiempo de dedicación.

- Incluir la información de contacto del bibliotecario en las asignaturas para facilitar la comunicación directa con los alumnos.

- Promocionar el servicio entre el profesorado.

Sobre los beneficios obtenidos, las bibliotecas que han implementado estos programas señalan una mejor valoración de la biblioteca por parte del profesorado, que toma consciencia de los recursos que les proporciona, un incremento en el uso de los recursos de la biblioteca (Cervera-Farré et al., 2015) y mayor rendimiento de la inversión institucional en costosos recursos bibliográficos (Cohen, 2002). Por otra parte, a medida que la tecnología mejora la experiencia de los estudiantes a distancia, quienes asisten a clases presenciales se benefician también de estos servicios virtuales (Behr; Hayward, 2016), ya que pueden utilizarlos desde cualquier lugar. Esto sucede porque el campus virtual no siempre es exclusivo del alumnado a distancia, sino que también se emplea como complemento a las asignaturas presenciales, como un espacio donde los docentes comparten bibliografía y otros recursos de apoyo para la enseñanza y se comunican con sus estudiantes. Además, se podría considerar como beneficio un mejor conocimiento y aprendizaje en lo relacionado con la alfabetización informacional por parte del alumnado, lo que revierte en una mejora de sus procesos de aprendizaje.

Cada vez son menos las barreras técnicas para la integración de la biblioteca en el campus virtual, debido a las mejoras tecnológicas, pero siguen existiendo dificultades de otro tipo, que resume Jackson (2007) ordenadas de mayor a menor relevancia:

- La relación entre el personal bibliotecario y el docente.

- Coste y tiempo invertidos por los bibliotecarios.

- La curva de aprendizaje del bibliotecario.

- La motivación de los estudiantes para usar los recursos.

A estas dificultades hay que sumar, al menos en el caso de las universidades españolas, las relaciones con otras áreas de la propia universidad, como pueden ser las responsables del campus virtual o los servicios informáticos.

Como complemento a la integración de la biblioteca en el campus virtual, resulta imprescindible elaborar un buen plan de 
comunicación de los servicios a distancia que oferta la biblioteca, que a menudo son desconocidos para los estudiantes hasta que los necesitan. En este sentido, Behr y Hayward (2016) señalan las deficiencias de los sitios web de las bibliotecas, que en ocasiones no recogen toda la información sobre estos servicios o no se encuentra lo suficientemente actualizada.

Las propuestas de integración de la biblioteca en el campus virtual no son nuevas. Cohen (2002) ya proponía enlaces a los recursos, bases de datos y catálogos, referencia virtual, contacto directo con el bibliotecario e incorporar los llamados course reserves - una colección de manuales reservados para los alumnos de determinadas asignaturas (Pollitz; Christie; Middleton, 2009)-, así como la necesidad de colaborar con el profesorado.

En la bibliografía en español se ha tratado este tema con menor frecuencia, pero tampoco resulta nuevo. Rebiun (2007), en su II Plan estratégico, ya tenía la visión de

"una biblioteca digital conectada con los campus virtuales de la universidad".

Se trataba de

"integrar la biblioteca digital en los campus virtuales de las universidades y potenciar el aprendizaje en red de nuestros estudiantes y profesores".

Esta visión ha tenido continuidad en el III Plan Estratégico (Rebiun, 2014), que señala en la línea 2 el objetivo de

"potenciar la biblioteca como un agente dinamizador de la innovación docente de la universidad e incrementar

el uso de los recursos de información desde las diferentes plataformas educativas virtuales de las universidades".

Por otra parte, Caridad-Sebastián y Martínez-Cardama (2013) recogieron varias de las formas de integración del bibliotecario en el campus virtual.

La figura del bibliotecario integrado tampoco es nueva en la bibliografía española; se ha tratado más bien como una figura genérica que sirve de enlace con las facultades y departamentos, sin diferenciar docencia o investigación, con varias experiencias que lo atestiguan (Martínez-Trujillo, 2013; Zapirain-Sagaseta, 2014). Más especializado en la docencia está el liaison librarian o bibliotecario enlace de la Universitat Oberta de Catalunya (Cervera-Farré et al., 2015).

A pesar de que estas propuestas vienen de hace tiempo, cobran ahora especial actualidad. Lutz y Schonfeld (2020) han recogido recientemente las impresiones de cuarenta directores de bibliotecas académicas, expresadas en varias mesas redondas organizadas por Ithaka $S+R$, una institución estadounidense sin ánimo de lucro que apoya a las universidades en el uso de la tecnología digital. En estas mesas redondas, en las que se debatía sobre los cambios que introduce la pandemia en las bibliotecas académicas, se pone de relieve el trabajo de apoyo a la docencia por parte de los bibliotecarios y el papel de los bibliotecarios integrados en el campus virtual, como enlace en el lugar donde están los estudiantes,

"creando roles de bibliotecarios en determinadas clases, desarrollando cuestionarios para estudiantes y proporcionando recursos electrónicos en el lugar donde son necesarios".

Además, se señalan otros puntos relevantes, como las colecciones digitales que se han venido desarrollando durante años, y la necesaria promoción de los recursos electrónicos.

En España, Rebiun (2020b) recomienda fomentar los servicios virtuales priorizando la adquisición de libros y revistas en formato digital, favoreciendo el préstamo electrónico, potenciando consultas y trámites online, consolidando la formación virtual, promoviendo la creación de materiales formativos y facilitando la digitalización de contenidos impresos.

\section{Apoyo a la docencia online en las bibliotecas universitarias españolas}

\subsection{Objetivos y metodología}

El objetivo de este trabajo es conocer las acciones que las bibliotecas universitarias españolas han llevado a cabo para adaptar al entorno digital sus servicios de apoyo al aprendizaje online, como consecuencia de la pandemia del Covid-19 que obligó al confinamiento social. Todo ello con especial atención a la integración en el campus virtual, el espacio en el que se encuentran los estudiantes de enseñanzas no presenciales.

El objetivo se desglosó en estos más específicos:

- Conocer las formas actuales de participación de las bibliotecas en el campus virtual.

- Identificar las mejoras necesarias en expresión del personal responsable de las bibliotecas.

- Conocer qué acciones externas al campus virtual están realizando las bibliotecas para complementar la enseñanza.

- Identificar cambios en las demandas de los usuarios con motivo de la virtualización de la enseñanza.

- Conocer qué limitaciones dificultan la mayor integración de las bibliotecas en el campus virtual.

- Recoger las propuestas que las bibliotecas realizan para su mejor inserción en el campus virtual a medio o largo plazo.

Para obtener la información de las acciones y servicios de las bibliotecas, aplicamos una metodología cualitativa, pues queríamos conocer lo más profundamente posible las dimensiones de un fenómeno muy complejo y en un momento casi simultáneo a los acontecimientos determinantes de su transformación. Esta metodología ha permitido realizar un primer análisis exploratorio y conocer cuál es la percepción de esta problemática por parte de las bibliotecas universitarias españolas participantes. 
La técnica cualitativa fue la entrevista, aplicada a una muestra de profesionales de bibliotecas universitarias españolas que tuvieran experiencia o responsabilidad en los servicios objeto del estudio. En la selección de los entrevistados y entrevistadas se aplicaron varios criterios para asegurar la representación de la diversidad de bibliotecas universitarias existentes, configurando así una muestra estratificada que asegurara la riqueza informativa. Para alcanzar esta heterogeneidad se tuvieron en cuenta las siguientes variables de clasificación:

a) tamaño (universidad con más de 30.000 estudiantes, entre 30 y 10.000 y menor de 10.000 estudiantes);

b) antigüedad (universidades históricas, existentes antes de 1975 y creadas ya en la España democrática);

c) estatus jurídico (universidades públicas o privadas);

d) formación ofrecida (generalista, especializada y politécnica);

e) modalidad docente (presencial o a distancia); y

f) contexto territorial (ubicación en diferentes comunidades autónomas).

Finalmente, las bibliotecas a las que se envió la entrevista y proporcionaron su respuesta fueron las indicadas en la tabla 1.

Tabla 1. Bibliotecas universitarias participantes en la entrevista

\begin{tabular}{|c|c|c|c|}
\hline Universidad & Titularidad & Modalidad de enseñanza & Territorio \\
\hline Universidad Complutense de Madrid & Pública & Presencial & Comunidad de Madrid \\
\hline Universidad de Castilla-La Mancha & Pública & Presencial & Castilla-La Mancha \\
\hline Universidad de León & Pública & Presencial & Castilla y León \\
\hline Universidad de Málaga & Pública & Presencial & Andalucía \\
\hline Universidad de Murcia & Pública & Presencial & Región de Murcia \\
\hline Universidad de Navarra & Privada & Presencial & Navarra \\
\hline Universidad de Salamanca & Pública & Presencial & Castilla y León \\
\hline Universidad de Valladolid & Pública & Presencial & Castilla y León \\
\hline Universitat Jaume I & Pública & Presencial & Comunidad Valenciana \\
\hline Universidad Nacional de Educación a Distancia (UNED) & Pública & A distancia & España \\
\hline Universitat Oberta de Catalunya (UOC) & Privada & Virtual & Cataluña \\
\hline Universitat Politècnica de Catalunya & Pública & Presencial & Cataluña \\
\hline Universitat Politècnica de València & Pública & Presencial & Comunidad Valenciana \\
\hline Universidad Pontificia de Salamanca & Privada & Presencial & Castilla y León \\
\hline
\end{tabular}

Para la entrevista, que administramos por correo electrónico a principios del mes de mayo de 2020 y se respondió hasta el día 15 de ese mismo mes, formulamos cinco preguntas, en correspondencia con los objetivos específicos, de las cuales la cuarta tuvo dos apartados, y la quinta tres. Fueron las siguientes:

1. ¿Cómo está presente la biblioteca en el campus virtual?

2. ¿Crees que podría mejorar la presencia de la biblioteca en el campus virtual de alguna manera? ¿De qué forma?

3. ¿Qué otras acciones de apoyo a la enseñanza online se están llevando a cabo fuera del campus virtual durante estos días por parte de la biblioteca?

4. ¿Has observado cambios en las demandas, el acceso y uso de la biblioteca por parte de profesorado y estudiantes durante el período de confinamiento, en relación con su actividad de enseñanza y aprendizaje?

5. ¿Crees que los actuales servicios digitales que ofrece la biblioteca son suficientes para cubrir las necesidades del alumnado respecto a la formación online a medio o largo plazo? (5.1) ¿Qué limitaciones observas? (5.2), ¿Cómo crees que podrían mejorarse? (5.3).

Una vez obtenidas las respuestas, realizamos un análisis de su contenido, y para ello se agruparon en tablas con las correspondientes a cada una de sus cuestiones. Cada una de las contestaciones se identificó para su posible mención con un número correspondiente a cada biblioteca participante, aleatoriamente asignado para garantizar el anonimato de quienes las expresaron. A su vez, las respuestas a cada pregunta las agrupamos en torno a categorías de análisis, en función de aspectos como el grado de participación de la biblioteca en el campus virtual, la tipología de acciones y servicios para desarrollar esta presencia, la percepción sobre el grado de suficiencia y adecuación de las mismas, el rango de limitaciones y propuestas de mejora, etcétera. Ello permitió ordenar la presentación de los resultados, que se ejemplifican con la cita literal o verbatim de algunas de las personas entrevistadas. Los resultados dieron lugar finalmente a las conclusiones, mediante un proceso comparativo entre los mismos, y de estos con la bibliografía revisada. 


\subsection{Resultados}

\subsubsection{Integración de las bibliotecas en el campus virtual}

Entre las bibliotecas consultadas se han encontrado distintas modalidades de integración de la biblioteca en el campus virtual. Los enlaces en la bibliografía recomendada son la forma más habitual entre las bibliotecas entrevistadas, mientras que solo algunas incluyen enlaces en el campus virtual a la web de la biblioteca. Además, hay bibliotecas con hipervínculos a los recursos de la biblioteca, como plataformas o bases de datos específicas, guías temáticas o materiales de los OpenCourseWare. Sólo en un caso se menciona la integración de la herramienta de búsqueda.

De las bibliotecas entrevistadas, la de la Universitat Oberta de Catalunya es la que muestra un mayor grado de integración en la plataforma de enseñanza online, debido a su naturaleza digital y a su larga trayectoria. Incluso cuenta con un programa de bibliotecarios integrados en el aprendizaje (Cervera-Farré et al., 2015) y proporciona a sus docentes un conjunto de recursos de aprendizaje que han sido creados o gestionados por la propia biblioteca.

"En el Campus, la biblioteca actúa con otros servicios de la universidad de manera transparente, sin que el usuario sepa que está consultando la biblioteca. El ejemplo más típico son los más de 35.000 recursos de aprendizaje que los estudiantes hallan en sus aulas". (B6)

En algunos casos, las bibliotecas imparten formación a través del campus virtual. Se trata de formación específica sobre los recursos de la biblioteca, destinada a la elaboración de trabajos fin de grado, formación complementaria para estudiantes de las escuelas internacionales de doctorado y para el propio profesorado, más relacionada con la investigación. En algunas universidades esta formación incluye reconocimiento de créditos. Entre las respuestas recibidas, solo la UNED menciona explícitamente la participación en asignaturas de grados y masters, como recoge la bibliografía anglosajona (ACRL, 2016).

Finalmente, también hay bibliotecas universitarias que no logran estar presentes en el campus virtual, que incluso carecen de un enlace básico a la biblioteca y ni siquiera tienen acceso a él.

“En el campus virtual no hay ningún enlace a la biblioteca universitaria, pero supongo que los profesores, o por lo menos algunos, sí enlazarán con la parte de la biblioteca que les interese mostrar a sus alumnos. Nosotros no podemos acceder y no lo sé con seguridad". (B11)

"Se intentó durante mucho tiempo que pusieran el enlace a la biblioteca desde el Aula virtual y mejorar el acceso desde la bibliografía recomendada en la guía docente [pero] nunca lo consiguió". (B3)

\subsubsection{Mejoras para la integración de las bibliotecas en el campus virtual}

Todas las bibliotecas consultadas creen que se puede mejorar de alguna manera la integración de la biblioteca en el campus virtual, a excepción de dos. Una de ellas considera haber alcanzado la idoneidad de sus servicios en el entorno digital y no formula mejoras adicionales. La otra cree que ha desarrollado una notable variedad de servicios y duda de la posibilidad de abordar nuevas mejoras.

"Se podría mejorar, otra cosa es la capacidad de ir a más con respecto a lo que ya tenemos". (B2)

La gran cantidad de propuestas de mejora evidencian las carencias que las bibliotecas consideran tener en su apoyo a esta modalidad de aprendizaje, así como su voluntad de avanzar. Las bibliotecas más desconectadas del campus virtual comienzan reclamando que haya un enlace desde éste a la biblioteca y sus recursos, comenzando por la bibliografía recomendada si aún no está enlazada. Este primer paso debe continuarse con herramientas de comunicación directa con los usuarios, incluyendo la posibilidad de contar con recomendaciones sociales.

"Sería deseable ofrecer un servicio de atención bibliotecaria al estudiante también integrado en la plataforma de aprendizaje". (B12)

También se expresa la voluntad de incrementar la participación mediante acciones formativas de la biblioteca a través del campus virtual, si bien en alguna ocasión se señala un éxito insuficiente.

"Podríamos tener más oferta formativa. Aunque la demanda de los cursos en Moodle, que ya llevábamos haciendo varios años, no tiene siempre mucha demanda". (B11)

Las bibliotecas que ya tienen servicios amplios en el campus virtual se plantean trabajar en mejoras sustanciales:

"Estamos definiendo el rol de "observador" en el entorno virtual de aprendizaje, algo parecido al embedded librarian que aplicamos en el caso del investigador. $Y$ un portal del docente a imagen del portal del investigador en donde se incluye la actividad docente: exámenes, materiales, presentaciones, enlaces o vídeos". (B7)

"Estamos evaluando diferentes aplicaciones que permiten al docente importar contenidos de forma transparente desde nuestra herramienta de descubrimiento". (B7)

Por último, entre las reflexiones de mejora más globales o conceptuales siempre se demanda la mayor valoración de la función de la biblioteca en el conjunto de misiones de la universidad y se realza la cooperación de la biblioteca con otros servicios dependientes de vicerrectorados relacionados con la innovación pedagógica, la tecnología y la investigación. 
"Estamos trabajando para lograr hacernos un sitio en el espacio de la Universidad virtual; y ejemplo de este avance es que, desde hace algún tiempo, ya colaboramos con distintos Vicerrectorados". (B9)

"Incorporar a la biblioteca como imprescindible en la estrategia digital de la universidad". (B13)

"Esta mejora tendría que venir dada desde el reconocimiento, por parte de la Universidad, del relevante papel que tiene la Biblioteca como facilitadora de servicios de información y formación en un espacio virtual, y no solo en un espacio físico". (B9)

\subsubsection{Acciones de apoyo a la enseñanza online externas al campus virtual}

Las bibliotecas universitarias están desarrollando numerosas acciones de apoyo a la enseñanza online fuera del campus virtual. Aunque no sea algo generalizado, la mayor parte de ellas está desarrollando actuaciones de formación virtual sobre los recursos de la biblioteca para docentes y alumnos, algunas a través de videoconferencia. Una de las bibliotecas entrevistadas ha impartido además talleres gratuitos online para luchar contra la desinformación y la detección de bulos.

"Como experiencia piloto las bibliotecas han acometido la elaboración de una serie de cursos online por medio de Google Meet". (B4)

"Para teletrabajar, seguir clases, hacer deporte, estar en contacto con nuestros seres queridos, combatir el aburrimiento, comprar, necesitamos actualmente la mediación tecnológica. Por ello hemos desarrollado un mayor número de acciones". (B12)

"Otra pandemia que estamos viviendo y que se ha visto reforzada, si cabe, con la Covid-19 es la de las noticias falsas. Por ello, desde la biblioteca hemos iniciado múltiples labores para luchar contra la desinformación y la detección de bulos. Así, durante este confinamiento y en esta línea hemos organizado dos talleres gratuitos online para la comunidad universitaria para mejorar sus habilidades de detección de noticias falsas y verificación de contenido online". (B12)

Algunas bibliotecas han intensificado los servicios de comunicación digitales, atendiendo consultas a través de correo, redes sociales, WhatsApp, videollamadas o chat. Además, se trabaja en la elaboración de contenidos de formación, culturales y de apoyo, a través de diferentes formatos, como selecciones de recursos, vídeos, infografías o guías temáticas.

"Se está ofreciendo mucho apoyo a través de las redes sociales, contestando preguntas, lanzando vídeos sobre el uso de nuestros recursos y servicios. Los canales de comunicación en los que más nos han solicitado información han sido el correo electrónico y el número de WhatsApp de la biblioteca, que nos ha permitido entre otras cosas solucionar problemas a través de las llamadas y videollamadas de la aplicación, generando una sensación de atención y cercanía con los usuarios". (B10)

"Se ha creado una web específica para estudiantes, que informa periódicamente de los recursos en abierto y las sesiones de formación que ofrecen las editoriales estas semanas". (B14)

El refuerzo de las colecciones digitales se ha convertido en un punto clave, por lo que algunas bibliotecas están revisando la disponibilidad en formato digital de la bibliografía recomendada en las asignaturas y el incremento en la adquisición de contenidos en formatos digitales. Por otra parte, se han prestado portátiles a los usuarios que no disponían de ellos y prorrogado los préstamos para que no quedaran sobrepasados los plazos.

"Estamos invirtiendo el tiempo y los recursos económicos en resolver las lagunas que existen online, trabajando con el PDI en la revisión de bibliografías recomendadas para que tengan recursos que podamos ofrecer online, estamos prestando ordenadores de nueva adquisición, enviándolos a los domicilios de los estudiantes, etc.". (B1)

También se debería destacar, por su carácter colaborativo, la participación de las bibliotecas de la Universitat Oberta de Catalunya y la UNED en un proyecto denominado Conectad@s \#LaUniversidadEnCasa, alojado bajo el dominio de la UNED, que ha tenido como fin ayudar al conjunto del sistema universitario, a estudiantes, profesorado y la sociedad en general, facilitando acceso a recursos de aprendizaje en acceso abierto y a un conjunto de materiales formativos para la mejora de las competencias digitales.

\subsubsection{Cambios en las demandas de estudiantado y profesorado}

El brusco cambio de las condiciones de enseñanza ha supuesto una obligada transformación en estudiantado y profesorado, que les ha generado incertidumbre, con la preocupación principal para ambos de lo que supondrá la evaluación online y cómo se desarrollará.

"Las primeras dos semanas del confinamiento se han caracterizado por ser un momento de grandes cambios en el que alumnos y profesores han estado organizándose ante la nueva situación de enseñanza a distancia". (B5)

"Los estudiantes están muy descontentos, en general, con la incertidumbre de la evaluación final, con la forma de dar las clases online en algunos profesores, etc. Es lo que les preocupa más, la docencia". (B11)

"Saturación del alumnado con la novedad de la enseñanza virtual plena y la adaptación de los docentes". (B2)

Como consecuencia del cambio imprevisto y repentino en la docencia, las bibliotecas han detectado algunos cambios en las demandas por parte de profesores y alumnos. Casi todas las bibliotecas entrevistadas han experimentado un mayor 
uso de los recursos online y, como consecuencia, algunas observan más consultas sobre las formas de acceso y más peticiones de compra.

"Se ha incrementado enormemente el uso de recursos electrónicos poniendo de manifiesto el desconocimiento de las normas y procedimientos de acceso. En un pequeño porcentaje nos han seguido pidiendo documentos impresos, y en estos casos se ha ofrecido alternativas de documentos electrónicos". (B4)

"Preguntan cuándo se va a abrir la biblioteca para poder prestar libros impresos porque no les llega con el material digital que encuentran". (B10)

"Poco a poco han ido incrementándose las peticiones de compra online o de digitalización de artículos de nuestra colección impresa". (B5)

También sucede al contrario: se observa un descenso en los accesos a la web de la biblioteca porque ya no se pueden realizar en ella acciones habituales que tienen que ver con el uso de las instalaciones.

"Por otro lado, la actividad de nuestro portal ha descendido ya que las acciones más habituales son la reserva de salas, de equipos y horarios". (B7)

Otras demandas son el préstamo de ordenadores para estudiantes sin los medios tecnológicos necesarios y la asistencia en el uso de las herramientas básicas para el teletrabajo por parte del profesorado.

"Nos llamaron a las bibliotecas para ceder "sine die" nuestros portátiles de préstamo. Prácticamente todos los portátiles que tenemos han salido, de forma excepcional, durante la cuarentena". (B11)

"Han contactado con nosotros no solo para recibir asesoramiento en lo que a los recursos electrónicos se refiere, sino que del colectivo de docentes tenemos una mayor demanda de asistencia con la tecnología". (B12)

Finalmente, también hay hábitos que parecen mantenerse. El personal de las bibliotecas observa que el alumnado no valora suficientemente las competencias digitales, lo que condiciona las formas de aprender y de acceder a los recursos de la biblioteca.

"No se dan cuenta de la importancia de la adecuada gestión de la información hasta que tienen que hacer el trabajo de fin de grado y esta habilidad no es algo que se pueda adquirir con un curso en el segundo cuatrimestre del último curso". (B3)

\subsubsection{Limitaciones en el apoyo a la enseñanza online}

Una buena parte de las bibliotecas son conscientes de que sus servicios son insuficientes para una enseñanza virtualizada, pues no están bien integradas en el campus virtual ni en la estrategia digital de la universidad.

"Mi percepción es que la biblioteca está pasando la cuarentena, no sé si desapercibida, pero sí sin pena ni gloria". (B11)

"El tema es más complejo, no solo afecta a los servicios de la biblioteca, sino al resto de servicios de la universidad". (B7)

Las limitaciones se apuntan en cinco direcciones: las colecciones, cuestiones técnicas relacionadas con las plataformas de enseñanza, adaptación a la enseñanza online, aspectos relacionados con el personal y los presupuestos.

La oferta de colecciones digitales es una de las principales limitaciones con las que se encuentran las bibliotecas en el apoyo a la docencia online, especialmente en las áreas de humanidades y ciencias sociales. Ante la imposibilidad de acceder a los manuales impresos con las bibliotecas cerradas, es necesario reforzar las colecciones digitales. Sin embargo, sólo una parte de los títulos que integran la bibliografía recomendada de las asignaturas está disponible en formato digital.

“En el estado actual de la revisión observamos que según la titulación la disponibilidad digital de la bibliografía oscila entre el $17 \%$ y el $35 \%$. Suponemos que una vez realizada la revisión estos porcentajes serán más elevados". (B7)

A ello se suman otras limitaciones propias de los contenidos digitales, como la existencia de licencias que permitan el préstamo en bibliotecas y unos precios que se vuelven prohibitivos para dar acceso a una gran cantidad de usuarios. Las limitaciones son aún mucho mayores en el caso de los formatos audiovisuales, explica una de las bibliotecas consultadas.

Por otra parte, el profesorado no atiende a criterios de formato a la hora de elaborar la bibliografía recomendada de sus asignaturas, pero en algunas disciplinas sigue pesando más el formato impreso, debido a los propios hábitos de lectura del profesorado. Por ello es necesario orientar sobre la preferencia por los formatos digitales para facilitar el acceso.

Otras limitaciones están relacionadas con las cuestiones técnicas de las propias plataformas de enseñanza online y con los sistemas de búsqueda y recuperación de la información.

"La mayor parte de los campus virtuales tienen bastantes limitaciones y vienen a convertirse en poco más que repositorios de apuntes y/o documentos que no deberían estar allí, sino, en todo caso, enlazados y accesibles mediante las correspondientes contraseñas. [...] Las limitaciones principales son las técnicas y el soporte informático, pero tengo poca esperanza que en las actuales circunstancias se puedan mejorar. Tendremos que investigar las alternativas que nos permitan ser los más autónomos posible". (B4) 
Pero también se recogen otras cuestiones relacionadas con aspectos presupuestarios y de personal, como la carga de trabajo que supone la gestión de la crisis, la falta de personal y los recortes presupuestarios.

\subsubsection{Mejoras en los servicios online de las bibliotecas}

De forma general, todas las bibliotecas consultadas creen que los recursos y servicios que ofertan las bibliotecas para la enseñanza online actualmente se pueden mejorar, incluso algunos afirman que son insuficientes.

En cuanto a las mejoras por acometer, varias bibliotecas señalan la necesidad de mejorar la difusión, siendo este el aspecto en el que hay más coincidencia en las respuestas. Sin duda, el desconocimiento de las colecciones electrónicas por parte de los alumnos es uno de los obstáculos para su consulta. La encuesta bianual a alumnos de grado llevada a cabo por el Servicio de Bibliotecas de la Universidad de Navarra en 2018 desvelaba que un 50,7\% de los alumnos de grado desconocían la disponibilidad de libros electrónicos en la colección de la biblioteca (Servicio de Bibliotecas, 2018). Pero este desconocimiento también es habitual entre los profesores.

"Si los profesores no saben lo que tenemos, ¿cómo lo van a recomendar a los alumnos?" (B11)

Además se identifica una gran diversidad de mejoras: en la comunicación con el alumno a través de servicios como el chat, la integración de la formación virtual de la biblioteca en los currículos, sobre todo en alfabetización informacional, la creación de contenidos de formación, el sitio web y los servicios virtuales en general. Pero además, se pone de relieve la necesidad de ofertar servicios de calidad.

"Necesitamos reforzar la colección digital, dentro de esta colección resultan de gran interés los contenidos de las editoriales universitarias españolas". (B8)

"Es difícil crear materiales de formación, online o en persona, aunque la gente piensa que es fácil". (B4)

Todo ello requiere incrementar la colaboración dentro de la universidad para que la biblioteca esté más presente. Varias de las bibliotecas entrevistadas apuntan además que será necesario un tiempo de adaptación a la nueva situación.

"La Covid-19 ha supuesto un acelerador imprevisto para la transformación digital de la universidad. En el caso específico de [nuestra universidad] ha sido más sencillo afrontar el momento por nuestro sistema de aprendizaje a distancia". (B12)

"Tenemos que buscar más canales de comunicación dentro de la comunidad universitaria. No solo online, sino participación presencial, cuando se restablezca la normalidad, colaboraciones en diversos ámbitos de la vida universitaria para que la biblioteca esté más presente". (B11)

\section{Conclusiones}

Las bibliotecas universitarias españolas están trabajando para ampliar y mejorar sus servicios digitales de apoyo a la docencia y el aprendizaje, ante la perspectiva de un nuevo curso académico con un mayor componente virtual en la enseñanza, para el que se preparan las universidades. Prácticamente todas coinciden en la insuficiencia de los servicios actuales y en el amplio margen de mejora ante el nuevo escenario. Como consecuencia, todo apunta a que en el próximo curso veremos acelerar e intensificar este tipo de servicios digitales que ofertan las bibliotecas universitarias en España, sin que los servicios presenciales pierdan su valor ni su demanda, salvo por causas de fuerza mayor.

Los principales frentes de trabajo que se están acometiendo en este momento en el apoyo a la docencia y el aprendizaje online por parte de las bibliotecas consisten en:

- Facilitar el acceso online a la bibliografía recomendada por parte de los alumnos, siempre que sea posible.

- Trasladar la formación al entorno virtual, con sesiones a través de videoconferencia o del campus virtual y la elaboración de contenidos de apoyo.

- Ampliar la oferta de canales de comunicación digital con el usuario, como chat, WhatsApp o la asistencia a través de videoconferencia.

- Comunicar a alumnos y profesores los nuevos servicios de forma eficaz, para que tengan conocimiento de ellos y de cómo utilizarlos.

Sin embargo, en muchas bibliotecas universitarias está por abordar una mayor integración en el campus virtual de los recursos y del personal, de manera que los servicios bibliotecarios estén incluidos en el espacio donde están los alumnos online, al igual que otros recursos de aprendizaje. La biblioteca debe seguir siendo un componente importante dentro de la experiencia universitaria online para el alumnado, de la misma manera que lo es en el campus de la universidad, y la integración en las dinámicas de enseñanza virtual resulta esencial para ello.

En la actualidad, las bibliotecas se pueden clasificar en tres grupos en función del grado de integración en el campus virtual:

1. Nivel de integración bajo o nulo. Son bibliotecas que no están visibles en el campus virtual, ni siquiera mediante enlaces a la web de la biblioteca, pero que en algunos casos están empezando a trabajar en ello.

2. Nivel de integración intermedio. En este grupo se encuentran las bibliotecas que están visibles de alguna manera en el campus virtual, por ejemplo mediante enlaces a los recursos de la biblioteca y la bibliografía recomendada, o impartiendo algún tipo de formación. En este nivel se incluye la mayor parte de las bibliotecas consultadas. 
3. Nivel de integración alto. Es el caso de las bibliotecas que participan realmente en la enseñanza online dentro del propio campus virtual, debido a su modelo de enseñanza, y que tienen una trayectoria amplia en este sentido, por lo que su adaptación a la nueva situación no es necesaria.

Como era de esperar, las universidades puramente virtuales y con un modelo consolidado, como la Universitat Oberta de Catalunya, más cercano al de las bibliotecas norteamericanas, tienen una clara ventaja frente a las universidades presenciales en este momento y por ello se convierten en un punto de referencia obligado. Tras el análisis de la bibliografía internacional se extrae un gran número de experiencias a modo de referente. También se puede deducir que existe un amplio margen de mejora en la integración de las bibliotecas universitarias españolas en la enseñanza online.

Tanto los profesionales consultados como la bibliografía coinciden en la importancia de la colaboración de la biblioteca con los departamentos implicados en la docencia online y con el profesorado. Por lo tanto, la integración en la enseñanza online no consiste solo en enlazar a la web y a los recursos de la biblioteca, sino que además hay que definir estrategias claras y buscar alianzas, encontrar la manera de incluir a la biblioteca en las asignaturas de forma real y destinar recursos de personal para ello.

Las rígidas estructuras organizativas de algunas universidades y la falta de asociación entre la biblioteca y la innovación en las prácticas docentes suponen importantes barreras que las bibliotecas y las propias universidades deben superar. Por ello, las bibliotecas tienen el reto de demostrar lo que pueden aportar en el apoyo a la docencia y el aprendizaje online, más allá de ser espacios de estudio o facilitadoras de colecciones.

Las bibliotecas también se encuentran con limitaciones externas, como la escasez de contenidos digitales para el préstamo. En este sentido, es necesario requerir al mercado editorial una aceleración en la digitalización de contenidos y manuales para el alumnado, permitiendo su préstamo en bibliotecas en condiciones justas y equitativas y que permitan dar acceso a una gran cantidad de alumnos. En una situación como la actual, todos aquellos contenidos que no estén en formato digital para los alumnos serán prácticamente inservibles.

En un momento cambiante como éste es necesario establecer mecanismos permanentes de monitorización de las demandas. Las plataformas digitales proporcionan numerosos datos acerca del uso de los servicios y las colecciones online y permiten adaptarse a ellos.

En definitiva, se abre una interesante etapa en la evolución de las bibliotecas universitarias españolas sobre la que habrá que estar atentos. En esta etapa veremos acelerar el proceso de digitalización de los servicios bibliotecarios de forma inminente y cómo las bibliotecas se adaptan a la nueva situación. De esta adaptación y de las dinámicas de integración que sean capaces de construir dependerá su relevancia en la docencia y el aprendizaje online.

\section{Referencias}

ACRL (2016). Standards for distance learning library services. http://www.ala.org/acrl/standards/guidelinesdistancelearning

Behr, Michele D.; Hayward, Julie L. (2016). "Evolution or integration: What is the current state of library services for distance learners?". Journal of library and information services in distance learning, v. 10, n. 3, pp. 88-100. https://doi.org/10.1080/1533290X.2016.1206777

Black, Elizabeth L. (2008). "Toolkit approach to integrating library resources into the learning management system". The journal of academic librarianship, v. 34, n. 6, pp. 496-501.

https://doi.org/10.1016/j.acalib.2008.09.018

Caridad-Sebastián, Mercedes; Martínez-Cardama, Sara (2013). “El bibliotecario integrado en el aprendizaje universitario". El profesional de la información, v. 22, n. 2, pp. 149-154.

https://doi.org/10.3145/epi.2013.mar.09

Cervera-Farré, Albert; Cervera-Biedma, Elisabet; López-Pérez, Cristina; Santos-Hermosa, Gema; Vaquer-Suñer, Cristina (2015). "Hacia el liaison librarian: transformación de servicios bibliotecarios para dar apoyo a la docencia en la Universitat Oberta de Catalunya (UOC)". El profesional de la información, v. 24, n. 2, pp. 121-130.

https://doi.org/10.3145/epi.2015.mar.05

Clossen, Amanda (ed.) (2018). "Integrating the library in the learning management system". Library technology reports, v. 54, n. 5.

https://doi.org/10.5860/ltr.54n5

Cohen, David (2002). “Course-management software: where's the library?”. Educause, v. 37, n. 3, pp. 12-13.

https://er.educause.edu/-/media/files/article-downloads/erm0239.pdf

Dans, Enrique (2020). "La enseñanza online ya no es una opción". Blog de Enrique Dans, 12 abril. https://www.enriquedans.com/2020/04/la-ensenanza-online-ya-no-es-una-opcion.html 
Farkas, Meredith G. (2015). "Libraries in the learning management system". Instructional technologies tips and trends. https://acrl.ala.org/IS/wp-content/uploads/2014/05/summer2015.pdf

Henry, Jo (2014). “Academic library liaison programs: four case studies”. Library review, v. 61, n. 7, pp. 485-496. https://doi.org/10.1108/00242531211288236

Hoffman, Starr; Ramin, Lilly (2010). "Best practices for librarians embedded in online courses". Public services quarterly, v. 6, n. 2-3, pp. 292-305. https://doi.org/10.1080/15228959.2010.497743

Jackson, Pamela A. (2007). "Integrating information literacy into blackboard: building campus partnerships for successful student learning". Journal of academic librarianship, v. 33, n. 4, pp. 454-461.

https://doi.org/10.1016/j.acalib.2007.03.010

Johnson, Larry; Adams-Becker, Samantha; Estrada, Victoria; Freeman, Alex (2014). NMC Horizon report: 2014 library edition. Austin, Texas: The New Media Consortium.

https://library.educause.edu/resources/2014/8/horizon-report-2014-library-edition

Kampa, Raj Kishor (2017). "Bridging the gap: integrating the library into Moodle learning management system a study". Library hi tech news, v. 34, n. 4.

https://doi.org/10.1108/LHTN-11-2016-0055

Karplus, Susan S. (2006). "Integrating academy library resources and learning management systems: The library blackboard site". Education libraries, v. 29, n. 1.

https://educationlibraries.mcgill.ca/article/view/219

Lutz, Kimberly D.; Schonfeld, Roger C. (2020). "Leading a library today. How library directors are approaching the challenges of the current moment". Ithaka $S+R$ blog, 30 April.

https://sr.ithaka.org/blog/leading-a-library-today

Martínez-Trujillo, Dídac (2013). El bibliotecario incrustado (the embedded librarian) en las bibliotecas de la Universitat Politècnica de Catalunya. Barcelona Tech.

http://hdl.handle.net/2117/18042

Piña, Anthony A. (2007). "Course management systems: overview and implications for libraries". Library hi tech news, v. 24, n. 5, pp. 7-9.

http://doi.org/10.1108/07419050710780335

Pollitz, John H.; Christie, Anne; Middleton, Cheryl (2009). "Management of library course reserves and the textbook affordability crisis". Journal of access services, v. 6, n. 4, pp. 459-484.

https://doi.org/10.1080/15367960903149268

Rebiun (2007). II Plan estratégico 2007-2010.

https://www.rebiun.org/sites/default/files/2017-11/II\%20Plan\%20Estratégico.pdf

Rebiun (2014). III Plan estratégico de Rebiun 2020.

https://www.rebiun.org/sites/default/files/2017-11/Plan\%20Estratégico\%20REBIUN.pdf

Rebiun (2020a). ¿Cómo están reaccionando las bibliotecas universitarias de Rebiun ante el Covid-19? https://www.rebiun.org/noticias/universidades/covid-19

Rebiun (2020b). Recomendaciones Rebiun para un protocolo sobre reapertura de las bibliotecas universitarias y científicas. http://hdl.handle.net/20.500.11967/510

Servicio de Bibliotecas (2018). Encuesta de satisfacción dirigida a alumnos de grado. Pamplona: Universidad de Navarra. https://hdl.handle.net/10171/53924

Shank, John; Dewald, Nancy-Hodge (2003). "Establishing our presence in courseware: Adding library services to virtual classroom". Information technology and libraries, v. 22, n. 1, pp. 38-43.

Torres-Salinas, Daniel (2011). “Integrados en la investigación: los embedded librarians”. Anuario ThinkEPI, v. 5, pp. $48-51$. https://recyt.fecyt.es/index.php/ThinkEPI/article/view/30466

York, Amy C.; Vance, Jason M. (2009). "Taking library instruction into the online classroom: Best practices for embedded librarians". Journal of library administration, v. 49, n. 1-2, pp. 197-209.

https://doi.org/10.1080/01930820802312995

Zapirain-Sagaseta, Pedro-José (2014). El bibliotecario temático de Comunicación de la Universidad de Navarra [Trabajo de fin de grado]. Universidad de León. Directora: Josefa Gallego-Lorenzo.

http://hdl.handle.net/10612/4017 